\title{
Probability model for data redundancy detection in sensor networks
}

\author{
Suman Kumar and Seung-Jong Park* \\ Computer Science Department and Center for Computation and Technology, \\ Louisiana State University, LA 70803, USA
}

\begin{abstract}
Sensor networks are made of autonomous devices that are able to collect, store, process and share data with other devices. Large sensor networks are often redundant in the sense that the measurements of some nodes can be substituted by other nodes with a certain degree of confidence. This spatial correlation results in wastage of link bandwidth and energy. In this paper, a model for two associated Poisson processes, through which sensors are distributed in a plane, is derived. A probability condition is established for data redundancy among closely located sensor nodes. The model generates a spatial bivariate Poisson process whose parameters depend on the parameters of the two individual Poisson processes and on the distance between the associated points. The proposed model helps in building efficient algorithms for data dissemination in the sensor network. A numerical example is provided investigating the advantage of this model.
\end{abstract}

Keywords: Sensor network, Bi-variate distribution, spatial correlation

\section{Introduction}

Sensor networks are growing in size, and future sensor networks will consist of hundreds to thousands of inexpensive wireless nodes, each with some computational power and sensing capability. One of the main characteristics of a typical sensor node is the limited power supply [11]. Sensing nodes typically exhibit limited capabilities in terms of processing, communication, and especially, power [5]. Under these constraint, it is very important to design and deploy sensor nodes strategically to maximize the life time of energy constrained sensor nodes. Different applications would have different constraints and priorities on how their sensor networks must behave $[6-8,10]$. Sending individual data items from sensors to sinks, it is more energy efficient to send aggregated data. The net effect of this aggregation [14] is considerable energy saving by transmitting less amount of data.

Since these sensors are densely deployed to detect common phenomena, it is expected that a high degree of spatial correlation will exist in the sensor network data. Correlations among the data representing an event are usually reflected in multiple measurements at different locations. This data correlation can exhibit temporally and spatially. On one hand, the high density of sensors gives several advantages like fault tolerance, reliability of data delivery, etc.; the other hand, it provides chanllenges for us to design energy efficient protocols. However, to achieve this we must first understand the spatial characteristics of sensor networks. This understanding indirectly would lead to the detection and the minimization of the amount of redundant data. The detection of redundant data directly is related to the nodes participating

\footnotetext{
${ }^{*}$ Corresponding author. E-mail: sjpark@csc.lsu.edu.
} 
in duplicate data delivery. Advantage of redundant data deliveries has also been exploited in wireless ad hoc networks $[2,9,19]$. Therefore, it is necessary to derive a mathematical model which can capture spatial correlation among nodes.

In this paper, we present and analyze the redundancy problem for wireless sensor networks. A model for redundant nodes is introduced. In this model, redundant data occur when the nearby sensor devices are separated by not more than twice their sensing radius. We adopt a bi-variate and associated Poisson distribution of sensor nodes. The nearest neighbor distribution for the distance between two nodes is obtained. The analysis and quantification of the level of redundancy would help in the design of QoS-related algorithms which would require the level of redundancy. The remainder of this paper is organized as follows. In Section 2, motivation and contribution behind this paper is discussed. In Section 3, we introduce our model for redundancy with a probabilistic expression dealing with near neighbor distribution. Section 4 investigates usefulness of such model by giving a numerical example. In Section 5, related works are provided in brief. The conclusion and future direction are discussed in Section 6.

\section{Motivation and main contribution}

\subsection{Motivation}

Densely deployed sensors would result in many sensors that would monitor some mutually common spatial region due to the overlapping of their individual sensing areas. Hence, the data reported by these sources would be correlated. To understand this, we have two sensors, sensor A and sensor B and suppose they are placed close enough so that their sensing regions are partially overlapping with each other. When sensor A and sensor B sends data, that data is spatially distributed on their whole sensing region. Hence, the overlapping area is sensed by both of the sensors, resulting in duplicate information. If this data is transferred to the sink, same data set from several sensors are observed by the sink. This results in wastage of transmission energy for sensors and the amount of information provided contains lots of duplicates. Hence, such correlations have to be eliminated as soon as possible. The existence of above mentioned spatial correlation resulting in data redundancy can have potential advantages for the development of efficient communication protocols well-suited for the WSN paradigm. For example, intuitively, due to the spatial correlation, data from spatially separated sensors is more useful to the sink than highly correlated data from nodes in proximity. Therefore, it may not be necessary for every sensor node to transmit its data to the sink; instead, a smaller number of sensor measurements might be adequate to communicate the event features to the sink within a certain reliability level. Similarly, for a certain event-tracking application, the measurement reporting frequency, at which the sensor nodes transmit their observations, can be adjusted such that temporally-correlated phenomena can be captured at the sink within a certain distortion level and with minimum energy-expenditure. To estimate such redundancy phenomena is necessary and is the main aim of this paper.

\subsection{Main contribution}

Although the estimation of redundancy has been studied by simulation [16], to the best of our knowledge, there is no paper presenting a analysis for such an estimation. In this paper, we introduce a heuristic model for data redundancy in spatially distributed sensor network to characterize the amount of redundancy existing among near neighbor nodes. For the general scenario, although in our analysis we 
introduce two different kind of sensor nodes (further referred as A and B), it does not affect the general analysis for uniform sensor node scenario. However, it may lead to useful result considering that there are at least two kinds of sensor nodes that differ in some sense ${ }^{1}$ and still lead to a simplified analysis. In this paper, we consider that whatever differences sensors have, they are distributed with the same master Poisson process. We recognize that the near neighbor distribution is the main factor contributing to the overlap of sensing regions among nodes that introduces data redundancy among sensor nodes. We give a probabilistic expression giving near node distribution and argue that for a given sensing range how many sensors can deliver partially redundant data

\section{System model}

In this section, we continue our discussion from the previous section and introduce our model for data redundancy in sensor network. As discussed earlier spatially overlapped region attracts the joint treatment of data to reduce the communication cost for the data of interest by exploiting the existing correlation. Continuing our two node scenario and assuming data is uniformly distributed throughout the spatial region, the data collected by some node $n_{i}$ in its sensing region $A_{i}$ is proportional to the sensing area. Hence, data sensed in area $A_{i}=\gamma A_{i}$ Where, $\gamma$ is some proportionality constant that depends on sensing ability of sensors. Hence, if two sensors $A$ and $B$ sensing the area $A_{r}$ and $B_{r}$ transmits the data to the third sensor, meaningful data is given as

$$
\gamma\left(A_{r} \bigcup B_{r}\right)
$$

and amount of redundant(duplicate) information is simply given as below assuming the source of redundancy caused by only overlapping sensing region of these two sensors

$$
\gamma\left(A_{r} \bigcap B_{r}\right)
$$

One figure of merit is correlation that measures the degree of redundancy which is the percentage of data that is varies with each other and for this case only, overlapping part of the sensing regions varies with each other. Hence, for sensing nodes $A$ and $B$, the correlation factor is given by

$$
\frac{A_{r} \bigcap B_{r}}{A_{r} \bigcup B_{r}}
$$

Assuming uniform node configuration of all the nodes, the sensing radius is $r_{s}$ and transmission range is $r_{t}$. the sensing area is given as $\pi r_{s}^{2}$.

For a particular node say $s$, all the other nodes in area $4 r_{s}^{2}$, shares some degree of redundant information with $s$. In Fig. 1, two nodes A and B has position vectors $r_{A}$ and $r_{B}$ respectively and $r_{s}$ is their sensing range, the condition for redundancy is given by

$$
\left|r_{A}-r_{B}\right|<2 r_{s}
$$

Hence, to quantify the redundancy for all the neighbors around a sensor node we have to find out its near neighbor distribution in its own sensing range. Our next section presents an analysis, assuming sensor nodes follows a spatial bivariate distribution for sensor nodes A and B. Here, we consider nodes A and B which are different in terms of sensing rate or some other figure of merit say sensing capability factor $\gamma$.

\footnotetext{
${ }^{1}$ Sensor Nodes can differ in sensing capability (introduces as factor $\gamma$ ) or those that differ in their sensing cycles which is the subject of future research or they can be entirely different(say optical and magnetic sensors).
} 


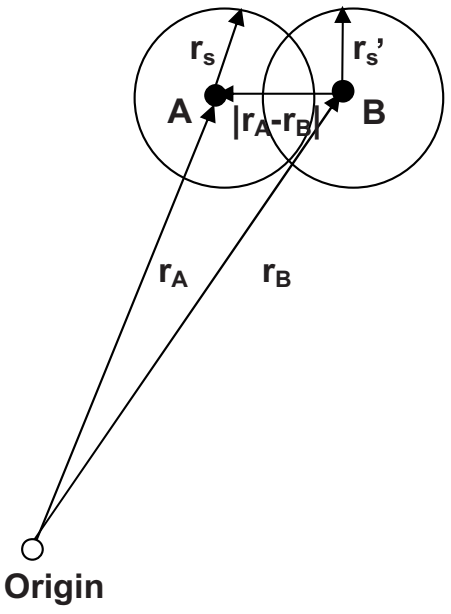

Fig. 1. Redundancy condition.

\subsection{Nearest neighbor distribution}

In this section, we present the near neighbor distribution considering the sensor nodes follow a bivariate spatial poisson point pattern [3,12]. First, the concept of bivariate distribution is presented in spatial sense and then an expression is derived representing the distribution of the distance from a sensor A to a nearest sensor B.

Maritz [15] obtained the probability generating function for the bivariate poison assuming that, in any interval of length $d t$, the combinations $(A \bar{B}, \bar{A} B, A B$ and $\overline{A B})$ of the two events $A$ and $B$, occur with probabilities $\lambda d t, v d t, \mu d t$ and $1-(\lambda+v+\mu) d t$. This model leads to probability generating function

$$
T(u, v)=\exp [\lambda(u-1)+\mu(v-1)+v(u v-1) t]
$$

The derivation of bivariate poisson distribution shows that the two events $A$ and $B$ are associated in some sense. This association can be described by the conditional probabilities

$$
\operatorname{prob}(A \mid B)=\frac{v}{\mu+v}, \operatorname{prob}(B \mid A)=\frac{\mu}{\mu+v}
$$

We may also consider that we have a process in time and events occur with a poisson density with parameter $(\lambda+v+\mu)$. Obviously the event is $A \bar{B}, \bar{A} B$, or $A B$ with probabilities respectively.

$$
\frac{\lambda}{\lambda+v+\mu}, \frac{\mu}{\lambda+v+\mu} \text { and } \frac{v}{\lambda+v+\mu}
$$

Given the probability generation function as above we have two near sensors representing events $A$ and $B$ are associated with the primary poisson process. The association between the two classes of events in this sense is peculiar in the sense that the two events, if they occur they occur together, occur simultaneously.

\subsubsection{Spatial bivariate distribution}

From the discussion from the previous section, we can generalize the Eq. (5) into a spatial distribution by simply replacing the $t$ with an area $s$. It would then be a probability generating function of the points 
of type $\mathrm{A}$ (sensor of one type) and $\mathrm{B}$ (sensor of another type) in an area of size $s$. We derive a spatial bivariate poisson process with density $\lambda+v+\mu$. Given a primary point $y=\left(y_{1}, y_{2}\right)$, with a probability $\frac{\lambda}{\lambda+v+\mu}$, it gives rise to a point of type A at some nearby point $x$. With probability $\frac{\nu}{\lambda+v+\mu}$, we have a point of type B at $x_{2}$ and with probability $\frac{v}{\lambda+v+\mu}$, we have a double point with A at $x_{1}$, and B at $x_{2}$. The locations $x_{1}$, and $x_{2}$, are determined so that the $\left(x_{1}-y\right)$ and $\left(x_{2}-y\right)$ are independent and have probability density functions $f\left(x_{1}-y\right)$ and $g\left(x_{2}-y\right)$, respectively.

Note that the same distribution is obtained from the model where, for a given primary point at $y$, there occurs a point A or B at $y$ with probabilities $\frac{\lambda}{\lambda+v+\mu}$ and $\frac{\nu}{\lambda+v+\mu}$, respectively, or then, with a probability $\frac{v}{\lambda+v+\mu}$, a double point with A at $x$, and B at $x$. The distances $\left(x_{1}-y\right)$ and $\left(x_{2}-y\right)$ are independent and have density functions $f\left(x_{1}-y\right)$ and $g\left(x_{2}-y\right)$. It now follows that

prob (a single point $\mathrm{A}$ in $\mathrm{dx})=\lambda d x$,

prob (a single point $\mathrm{B}$ in $\mathrm{dx})=\nu d x$,

prob (a double point with $\mathrm{A}$ in $d x$, and $\mathrm{B}$ in $d x$ )

$=\nu h\left(x_{1}, x_{2}\right) d x_{1} d x_{2}$

where

$$
h\left(x_{1}, x_{2}\right)=\int_{y \in R} f\left(x_{1}-y\right) g\left(x_{2}-y\right)
$$

and the integration is whole plane. Let $N_{1}\left(S_{1}\right)$ denote the number of points of type A in an area $S_{1}$ and $N_{2}\left(S_{2}\right)$ the number of points of type B in $S_{2}$. Since the marginal distribution of $A$ and $B$ are poisson and also only double points contribute to the covariance, we get from Eq. (8) for two different areas $S_{1}$ and $S_{2}$,

$$
\operatorname{cov}\left(N_{1}\left(S_{1}\right), N_{2}\left(S_{2}\right)\right)=\nu \int_{x_{1} \in S_{1}} \int_{x_{2} \in S_{2}} h\left(x_{1}, x_{2}\right) d x_{1} d x_{2}
$$

In the same line, analogous to Eq. (5) gives the probability generating function for the spatial bivariate Poisson distribution

$$
T(u, v)=\exp \left[\left(\lambda s+v\left(s-s^{\prime}\right)\right)(u-1)+\left(\mu s+v\left(s-s^{\prime}\right)\right)(v-1)+s^{\prime} v(u v-1)\right]
$$

For simplicity we define, $X_{A B}=$ Distance between A and B, and $r=$ Euclidean distance between two points $\mathrm{A}$ and $\mathrm{B}$. The distribution of the distance between two adjacent points, the nearest neighbor distribution considering marginal distributions are poisson, we get the following relationship

$$
\text { prob (distance from a point } \mathrm{B} \text { to next nearest point } B<r)=1-\exp \left(-(\mu+v) \pi r^{2}\right)
$$

and similarly for A. The distribution of the distance from a point A to a nearest point B may be derived as follows:

$$
\begin{aligned}
& \operatorname{prob}\left(X_{A B}>\mathrm{r}\right) \\
& =\operatorname{prob}(\text { A single }) \operatorname{prob}\left(X_{A B}>r \mid A \text { single }\right)+\operatorname{prob}(\text { A double }) \text { prob }\left(X_{A B}>r \mid A \text { double }\right) \\
& \quad=\frac{\lambda}{\lambda+v} \exp \left(-(\mu+v) \pi r^{2}\right)+\frac{\lambda}{\lambda+v} \exp \left(-(\mu+v) \pi r^{2}\right) \int_{|x|>r} h(x, 0) d x
\end{aligned}
$$

Hence,

$$
\operatorname{pr}\left(X_{A B}<r\right)=1-\exp \left(-(\mu+v) \pi r^{2}\right)\left(\lambda+v \frac{\int_{|x|>r} h(x, 0) d x}{(\lambda+v)}\right)
$$




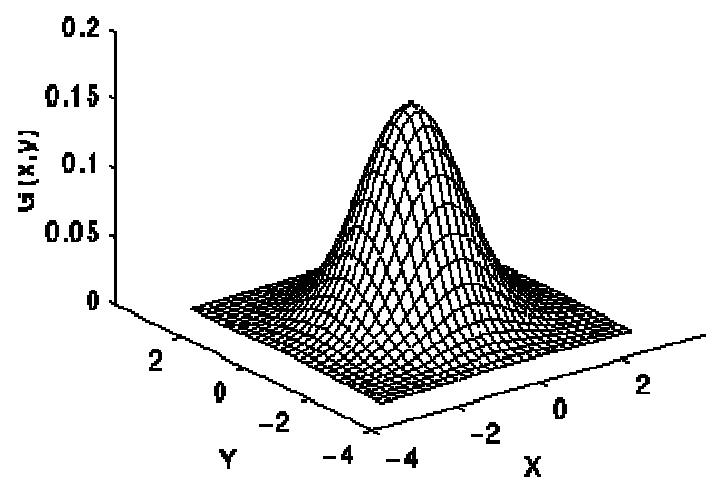

Fig. 2. 2-D Gaussian distribution with $\sigma=1$.

When $\mathrm{A}$ and $\mathrm{B}$ are independent, i.e. when $v=0$, Eq. (13) reduces to the distribution of the distance from a random point to the nearest point $B$ which is the same distribution as given in Eq. (11). To satisfy the condition of redundancy, we must have $X_{A B}<2 r_{s}$. Therefore, Eq. (13) is given as below:

$$
1-\exp \left(-(\mu+v) \pi 2 r_{s}^{2}\right)\left(\lambda+v \frac{\int_{|x|>2 r_{s}} h(x, 0) d x}{(\lambda+v)}\right)
$$

\section{A numerical example: An isotropic gaussian distribution}

In this section, we demonstrate the use of the formula we derived in the previous section. We take the case of isotropic Gaussian distribution. A circularly symmetric or isotropic Gaussian has the form:

$$
G(x, y)=\frac{1}{2 \pi \sigma^{2}} \exp \left(-\frac{1}{2}\left(\frac{x^{2}+y^{2}}{\sigma^{2}}\right)\right)
$$

and the distribution is shown in the Fig. 2. Let $f(\mathbf{x})$ and $g(\mathbf{x})$ be two isotropic gaussian distribution with variances $\sigma_{1}$ and $\sigma_{2}$ respectively. They are defined as below:

$$
\begin{aligned}
& f(\mathbf{x})=\frac{1}{\mathbf{2} \pi \sigma_{1}^{2}} \exp \left(-\frac{1}{2}\left(\frac{\mathbf{x}^{2}+\mathbf{y}^{2}}{\sigma^{2}{ }_{1}}\right)\right) \\
& g(\mathbf{x})=\frac{1}{\mathbf{2} \pi \sigma_{2}^{2}} \exp \left(-\frac{1}{2}\left(\frac{\mathbf{x}^{2}+\mathbf{y}^{2}}{\sigma_{2}^{2}}\right)\right)
\end{aligned}
$$

We have two position vectors $\mathbf{x}_{\mathbf{1}}$ and $\mathbf{x}_{\mathbf{2}}$ for point of type A and type B respectively. Hence, distance between the two is $\left(\mathbf{x}_{\mathbf{1}}-\mathbf{x}_{\mathbf{2}}\right)$. Then simple calculations show that

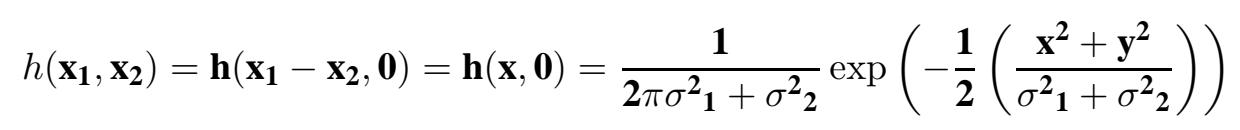

By substituting $h(\mathbf{x}, \mathbf{0})$ in Eq. (14) and integrating over the circular area $4 \pi r^{2}{ }_{s}$, we get

$$
\operatorname{pr}\left(X_{A B}<2 r_{s}\right)=1-\exp \left(-(\mu+v) \pi r_{s}^{2}\right)\left(\lambda+v \frac{\frac{1}{4} \sqrt{\frac{\pi}{a}}-\sqrt{\frac{1}{a}} \frac{1}{2} \sqrt{\pi} \operatorname{erf}\left(2 a r_{s}\right)}{\lambda+v}\right)
$$




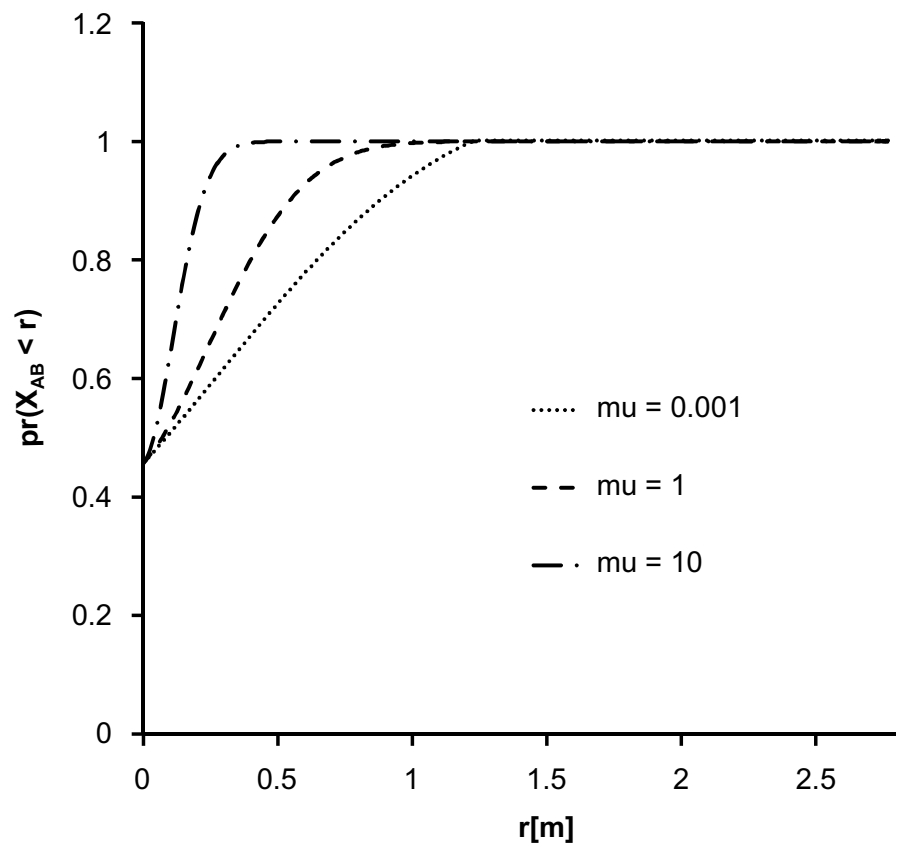

Fig. 3. $\operatorname{pr}\left(X_{A B}<r\right)$ variation with relative distance $\mathrm{r}$ for different $v(m u)$ values for $\lambda=0.1, \mu=0.1, \sigma_{1}=1$ and $\sigma_{2}=1$.

where,

$$
a=\frac{1}{2\left(\sigma_{1}^{2}+\sigma_{2}^{2}\right)}
$$

and $\operatorname{erf}(x)$ is the error function for each element of $x$ and given by:

$$
\operatorname{erf}(x)=\frac{2}{\operatorname{sqrt}(\pi)} X \text { integral from } 0 \text { to } x \text { of } \exp \left(-t^{2}\right) d t
$$

$v$ is related to the event that $\mathrm{A}$ and $\mathrm{B}$ both occurs i.e. the event type AB. Hence, we are interested in the variation of $\operatorname{pr}\left(X_{A B}<r\right)$ with radial distance r. In the Fig. 3, we show the probability variation for three values of $v$. As we can see, when $v$ is very low $(0.001)$, the $\operatorname{pr}\left(X_{A B}<r\right)$ is low and as we increase the value of $v$ the probability values increases. $v$ values plays an important role in nearest neighbor probability. For a densely distributed sensor network, $v$ value is large and hence results into more redundant data collected by neighborhood nodes.

Figure 4 shows the variation of $\operatorname{pr}\left(X_{A B}<r\right)$ with distance $r$ for different variance $(\sigma)$ values. As the value of variance increases, we expect less redundancy in the data values as shown in the figure. The figure demonstrates that variance is one critical design parameter. In both Figs 3 and 4, we see as distance increases, probability to find a nearest neighbor point increases and converge to 1 . However, not necessarily they all satisfy the condition of redundancy. Only those sensors fit the condition of redundancy that are separated by not more than sum of their sensing ranges.

There are different sensing ranges for different sensors. Under the given distribution, we can easily calculate the probability of two sensors overlapping each other's sensing ranges. We show the figures for only $3 \mathrm{~m}$ range as beyond that the probability converge to 1 (However, it may not be true for all the distributions) and remain constant for larger values of radial distance. 


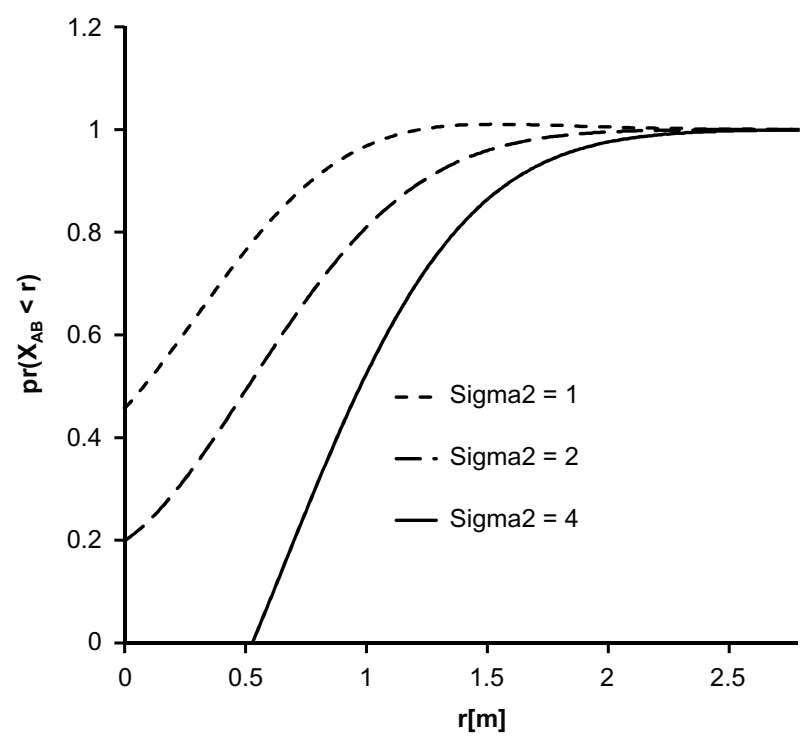

Fig. 4. $\operatorname{pr}\left(X_{A B}<r\right)$ variation with relative distance $\mathrm{r}$ for different $\sigma_{2}$ (Sigma2) values for $\lambda=0.1, \mu=0.1, v=0.1$ and $\sigma_{1}=1$.

\section{Related work}

In the literature, there has been work on covering a circle by arcs. Early in 1982, Siegel and Holst [17] studied the problem of covering a circle with random arcs of random sizes and established a complicated integral representation for the coverage probability.

[13] recognizes the negative effects of data redundancy and propose a Singular-Value-QR Decomposition method to reduce the data redundancy in wireless sensor networks. They first show that adjacent nodes generate similar data and establish the fact the redundancy exists among dense clusters of nodes. To reduce redundancy, they use the SVD method for subset selection and turn off other nodes. This paper is about reducing redundancy and not about estimating how much data redundancy is there in the network [18] analyzes the effect of redundancy on the mean time to failure of a network of wireless sensors in terms of energy-depletion. The authors show that there is a tradeoff between the degree of redundancy and the mean time to failure (MTTF). This paper deals with hardware redundancy and path redundancy to deal with sensor faults as against information redundancy and their estimation.

[4] has estimated the redundant sensing areas and have computed the minimum and maximum number of neighborhood sensors that are required to provide complete redundancy and have also formulated a method to estimate the degree of redundancy without the knowledge of location and directional information. The authors have focussed on the number of redundant nodes in the network as against our focus on the redundant data which could arise irrespective of the presence of redundant nodes. Though the authors have estimated the probability of complete redundancy with tight bounds, they have not directly dealt with the analysis of redundant data in the network and the different probabilities with which the individual sensors would transmit.

[1] studied the problem of detecting and eliminating redundancy in sensor networks with an intention to improve energy efficiency and maintain the coverage of the network. Their work also provides a study of the impact of eliminating redundancy on the coverage-boundary detection. They reduce these problems to the computation of Voronoi diagrams. Our paper is slightly different from this one in 
the sense that our paper estimates the data redundancy whereas, the paper by Bogdan et al. deals with redundant sensors and ways to eliminate them without affecting the coverage area.

\section{Conclusion}

Dense deployment of sensor network results into better operation using collaborative nature of wireless sensor networks. This collaboration results in redundant data which proved as a unique characteristic of a typical sensor network. In this paper, we introduced a redundancy model. In this model, redundant data occur when the nearby sensor devices are separated by not more than twice their sensing radius. Sensors are assumed to be distributed and follow a bivariate Poisson process. A probabilistic expression is found for near neighbor distribution. Also a numerical example is provided for a special case of isotropic Gaussian distribution. For simplicity, our model captures the bi-variate case, however it can be easily extended for multivariate case by including more diversity in sensor distribution in a given area. Also, it can be extended to three dimensional case by including the another space variable.

Our results will benefit the research on wireless sensor networks by providing simple estimation of redundancy to design new energy efficient protocols. Our further work will involve finding the distribution of overlap area and then give an estimation of redundancy using different distributions(including random) of sensor nodes. Further extension of this work also will involve analysis of sensor network for different variation and distribution of sensor nodes. We believe, the presented model may be unrealistic for concrete sensor technology which usually takes into account the sensor orientation, angular aperture, time-varying properties of detections (using different sleep and wake up cycles), etc. In this sense, the analytical results of this paper are only suitable for the situation where each node can persistently perceive phenomena within its surrounding circular area (as we assumed the uniform sensing radius, however it does not affect the generality of the formulation). Since it is very difficult to provide a general model that can capture the behavior of different sensors for different sensing tasks (say magnetic, optical etc.), we will expand our analysis to particular sensor technology and for various sensor network application scenarios.

\section{Acknowledgment}

This work was funded in part by BoR grant LEQSF(2006-08)-RD-A-08 and Dept. of Navy (Office of Naval Research) grant N00014-08-1-0856.

\section{References}

[1] B. Carbunar, A. Grama, J. Vitek and O. Carbunar, Redundancy and coverage detection in sensor networks, ACM Transactions on Sensor Networks (TOSN) 2(2) (February 2006).

[2] B. Chen, K. Jamieson, H. Balakrishnan and R. Morris, Span: An energy-efficient coordination algorithm for topology maintenance in ad hoc wireless networks, In Proceedings of the fifth annual international conference on Mobile computing and networking (MobiCom 99), pages 85-96, 1999.

[3] Peter J. Diggle, Spatio-temporal point processes: Methods and application, Monograph on Statistics and Applied Probability 107, Chapman and Hall/CRC, pages 1-45, 2007.

[4] Y. Gao, K. Wu and F. Li, Analysis on the redundancy of wireless sensor networks, In Wireless Sensor Networks and Applications, San Diego, CA, September 192003.

[5] G.J. Pottie and W.J. Kaiser, Wireless integrated network sensors, Communications of the ACM 43(5) (May 2000), 51-58. 
[6] Anna Hac, Wireless Sensor Network Designs, John Wiley and Sons, 2003.

[7] Y. Sankarasubramaniam, I.F. Akyildiz, W. Su and E. Cyirci, Wireless sensor networks: A survey, COMPUTER NETWORKS 38(4) (2002), 393-422.

[8] M. Ilyas and I. Mahgoub, Handbook of Sensor Networks - Compact Wireless and Wired Sensing Systems, CRC Press, 2004.

[9] S. Sitharama Iyenger and R.R. Brooks, Distributed Sensor Networks, Chapman and Hall/CRC publishing house, 2005.

[10] J. Kahn, R. Katz and K. Pister, Next century challenges: Mobile networking for smart dust, In Fifth annual international conference on Mobile computing and networking (MobiCom 99), pages 263-270, Seattle, USA, August 1999.

[11] J. Kahn, R. Katz and K. Pister, Next century challenges: Scalable coordination in sensor networks, Proceedings of International Conference on Mobile Computing and Networking (MobiCom), Seattle, USA, August 1999.

[12] H.-H. Kuo, Introduction to Stochastic Integration, Springer (Universitext), 2006.

[13] Q. Liang and L. Wang, Redundancy reduction in wireless sensor networks using svd-qr, Military Communications Conference, 2005. MILCOM 2005. IEEE, pages 1857-1861, Vienna, Austria, Oct 17-20 2005.

[14] S. Madden, M.J. Franklin, J.M. Hellerstein and W. Hong, Tag: a tiny aggregation service for ad-hoc sensor networks, Proceedings of the 5th Symposium on Operating Systems Design and Implementation 39 (2002), 131-146.

[15] J.S. Maritz, Note on a certain family of discrete distributions, Biometrika 39 (1952), 196-198.

[16] S. Ni, Y. Tseng, Y. Chen and J. Sheu, The broadcast storm problem in a mobile ad hoc network, MobiCom, pages 151-162, August 1999.

[17] A. Siegel and L. Holst, Covering the circle with random arcs of random sizes, Journal of Applied Probability 19 (1982), 373-381.

[18] A.P. Speer and I.-R. Chen, Effect of redundancy on mean time to failure of wireless sensor networks, In Proceedings of the 20th International Conference on Advanced Information Networking and Applications (AINA'O6), Vienna, Austria, April 18-20 2006.

[19] Y. Xu, J. Heidemann and D. Estrin, Geography-informed energy conservation for ad hoc routing, In Proceedings of the seventh annual international conference on Mobile computing and networking (MobiCom 01), pages 70-84, 2001.

Suman Kumar is a PhD candidate in the Computer Science Department and Center for Computation Technology at Louisiana State University. He recieved his B.Tech. degree in Electronics and Communication Engineering from the Indian Institute of Technology (IIT), BHU, India in 2003. During his B.Tech, he published a few research papers in international journals and conferences in solid state and microwave devices.His current research interests include High Speed Networks, Wireless Sensor Networks, Network Modelling and Simulation, High Performance Computing, and Algorithm Optimization.

Seung-Jong Park is an assistant professor in the Computer Science Department and Center for Computation Technology at Louisiana State University. He leads the LSU Advanced NETworking (LANET) group, where he and his students do research in the area of computer networking, such as wireless networks and high speed networks. He received his Ph.D. from The School of Electrical and Computer Engineering at Georgia Institute of Technology, 2004. Prior to that, he had also received a B.S. degree in Computer Science at Korea University, Seoul, Korea and a M.S. degree in Computer Science from KAIST (Korea Advanced Institute of Science and Technology), Teajon, Korea in 1993 and 1995, respectively. From 1995 to 2000, he had worked for Shinsegi Telecomm, which was the first CDMA cellular service provider in the world. 

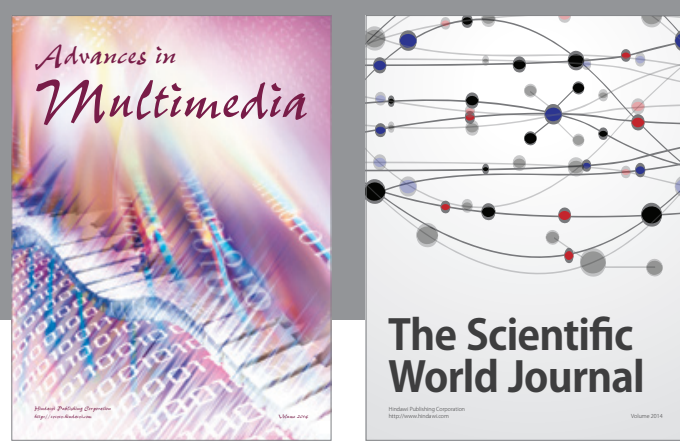

The Scientific World Journal
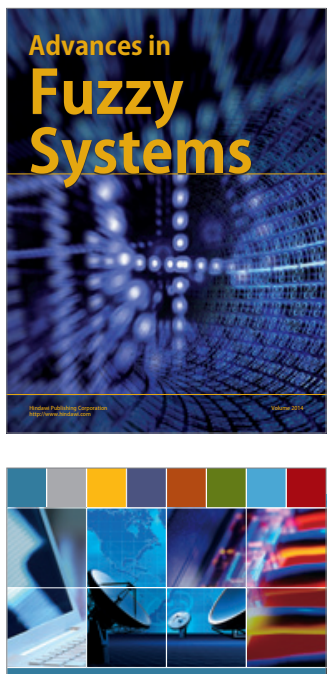

Computer Networks and Communications
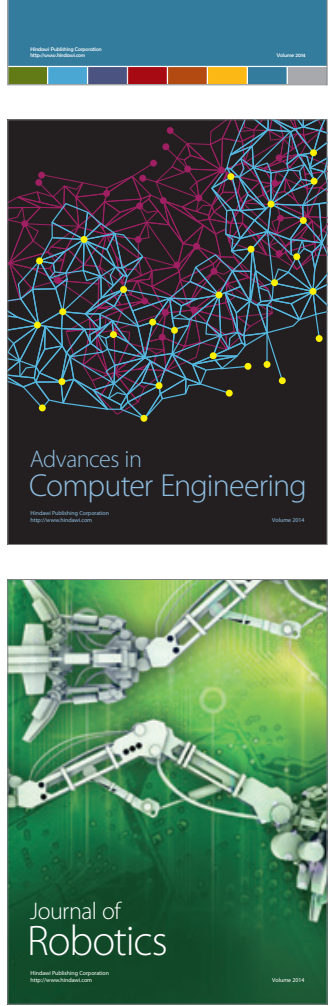
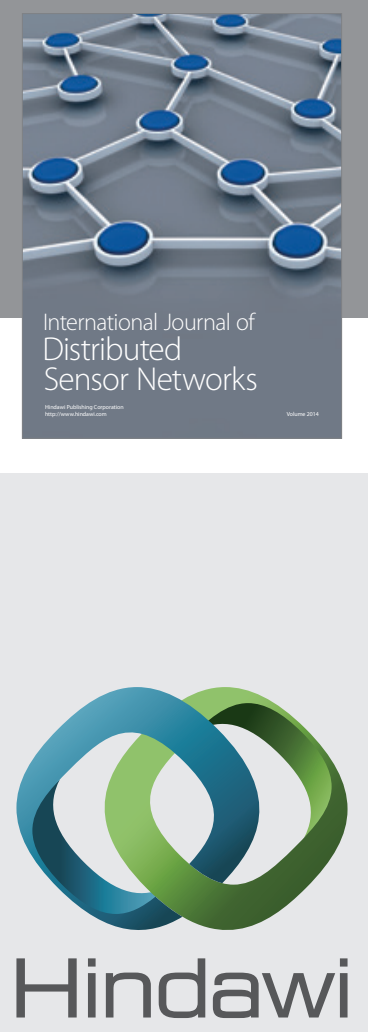

Submit your manuscripts at

http://www.hindawi.com
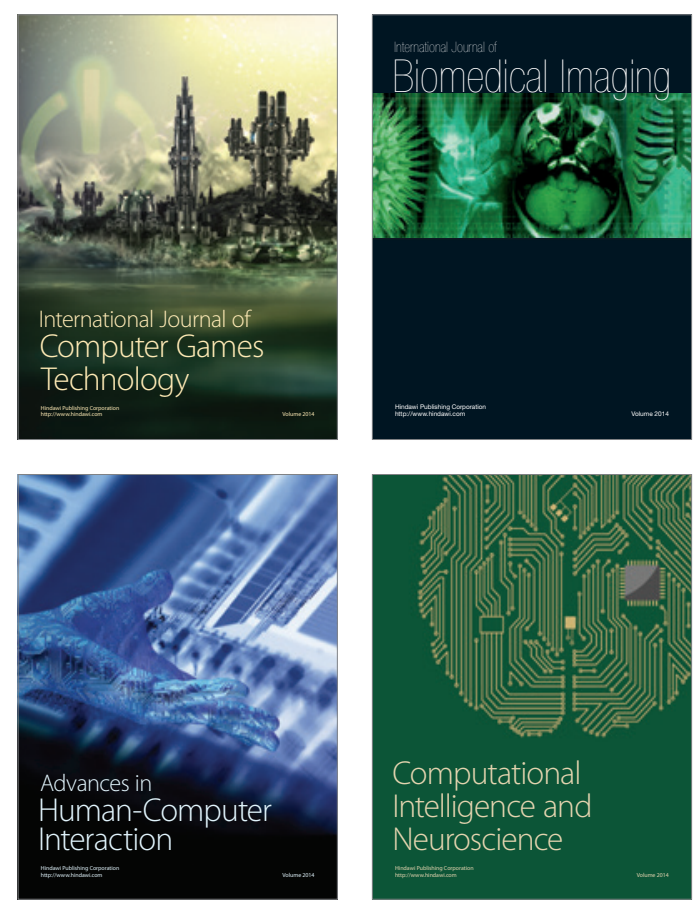
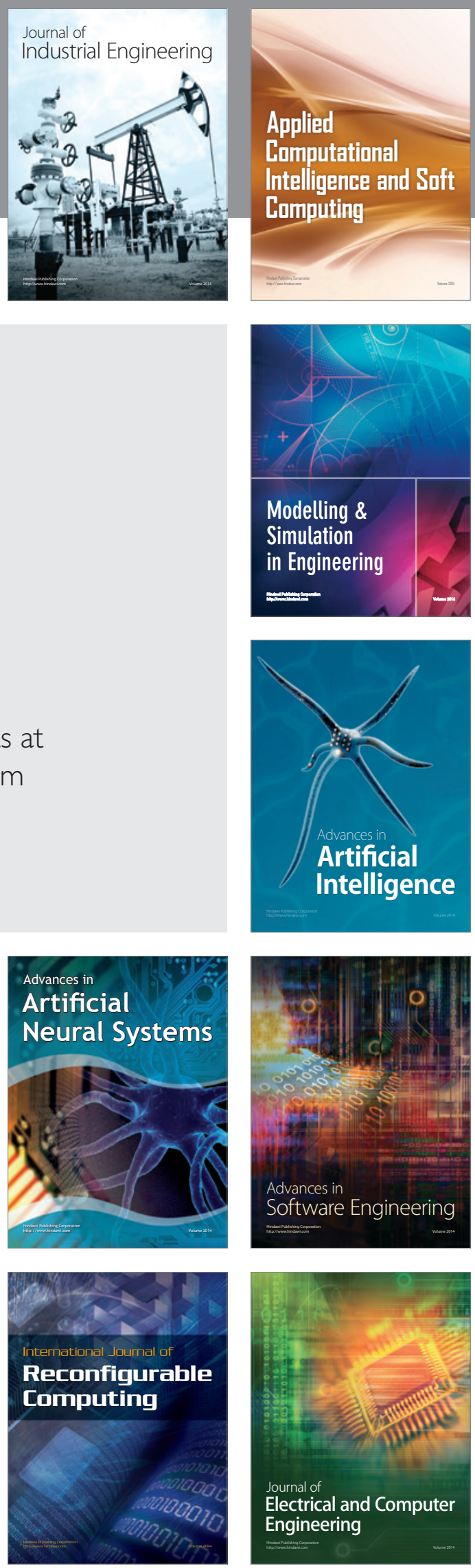